\title{
Development of a technique for predicting the human response to an emergency situation
}

\author{
Glyn Lawson ${ }^{1}$, Sarah Sharples ${ }^{1}$, David Clarke ${ }^{2}$, and Sue Cobb ${ }^{1}$ \\ ${ }^{1}$ Human Factors Research Group, Faculty of Engineering, The University of Nottingham, \\ United Kingdom, NG7 2RD \\ ${ }^{2}$ School of Psychology, The University of Nottingham, United Kingdom, NG7 2RD \\ \{glyn.lawson, sarah.sharples, david.clarke, sue.cobb\}@nottingham.ac.uk
}

\begin{abstract}
This paper presents development work on a new approach for predicting the human response to an emergency situation. The study builds upon an initial investigation in which 20 participants were asked to predict what actions they would take in the event of a domestic fire [1]. The development work involved a retest with an additional 20 participants to investigate the reliability of the approach. Furthermore, the analysis procedure was improved such that the results represented more accurately those which could be obtained from practical application of the approach. As found in the initial investigation, the frequencies and sequences of the reported acts had significant relationships with a study of behavior in real fires [2] (Spearman's rho: 0.323, N=55, $<<0.05$ ) and (Spearman's rho: 0.340, N=37, p<0.05), respectively. Further development work is required, but the results indicate that the approach may have use for predicting human behavior in emergencies.
\end{abstract}

Keywords: human response, behavior, emergency, reliability, predict

\section{Introduction}

Predictions of human behavior can be used by Human Factors (HF) professionals to analyze interaction with a system at an early stage in the development process. Predictions typically involve abstracting complex human behavior into models which are analyzed against the tasks the user will have to perform to achieve their desired goal on the planned system. This process can identify mismatches between human capabilities and system requirements, or quantify the predicted human performance. It can reduce the cost associated with identifying HF issues at a later stage in the development process, when physical prototypes become available for user trials, as design decisions become fixed and investment will have been made into specific lines of engineering [3].

Publications in the HF literature regarding predictions of human performance are typically concerned with nominal operating conditions, e.g. [4]. However, methods are also required for predicting behavior in emergency situations. These would have several applications: understanding the human response to an industrial incident during factory layout planning [5]; developing training plans for emergency response 
teams [6]; and improving the design of evacuation routes and guidance in buildings, aircraft and boats [7-9].

Predicting behavior in emergencies is required for reasons other than reducing development costs. Ethical considerations prevent putting participants in distress, and therefore the relevant behaviors cannot be studied in a laboratory, even if a physical prototype or the real system is available [10]. Basing predictions on previous events is not always possible, as gaining access to incident reports can be difficult. Furthermore, emergency events are unique to the particular circumstances; the validity may be questioned of a behavioral prediction derived from a different scenario [11].

This paper presents development work on a new approach for predicting human behavior in emergency situations. The approach is based on a talk-through method in which participants are asked to describe their actions in response to a scenario [12]. This low-cost approach means that a dangerous situation can be described without putting participants in any physical risk. The approach also draws upon sequential analysis [13], an established technique for studying dynamic aspects of behavior. Sequential analysis requires the assessor to record behaviors according to a taxonomy. Sequences of behavior can then be analyzed, for example by recording the number of times behavior $\mathrm{X}$ follows behavior $\mathrm{Y}$.

These methods were chosen in the initial development of this predictive approach [1] to enable comparability with sequential analysis data from a study of real behavior in domestic fires [2]. The comparison study [2] involved interviews with 41 people who had been involved with domestic fires, who were asked to describe exactly what they did from the time they noticed something abnormal was happening, until they reached a safe area. The descriptions were transcribed and coded using a taxonomy of behaviors. The frequency of acts, and act sequences, were analyzed and published.

The initial predictive study [1] demonstrated comparability of the frequency and sequence of a selection of reported actions with the study of behavior from people involved with real fires [2]. However, reliability is an important criterion for the success of a method or technique, which essentially requires that the same results are achieved upon repeated use of the method [14]. This investigation aimed to investigate reliability of the predictive approach through a test-retest approach, in which the methodology was replicated with different participants. It was also decided to investigate in further detail the validity of the predictive approach by removing from the analysis only two acts. These were related to technology which has become more prevalent since 1980. This differs to the analysis used in Lawson et al. [1], in which any categories not expected due to the example scenario or experimental protocol were removed. This change simplifies the administration of the approach as a predictive tool, as users are likely to find it difficult to identify which tasks to remove from the analysis unless justification is obvious, as it is with the two acts relating to a change in technology over several decades. 


\section{Method}

Adverts were places around the University of Nottingham, and were circulated by email. The adverts explained that research was being conducted into human behavior in emergencies. They told people not to apply if they had been involved (or had a close friend or relative who had been involved) in a fire, or if they suffered from any mental ill health. These precautions were put in place to avoid causing participants any distress. Participants from the initial investigation [1] were also excluded as the aim was to investigate whether the results drawn from a different sample would also demonstrate a significant relationship with the study of behavior in real fire [2].

20 participants ( 14 male, 6 female; mean age $=31.32, \mathrm{SD}=5.47)$ met the application criteria and were allocated a 1 hour appointment each. Each participant was asked to sign a consent form which emphasized that they could pull out at any point if they felt distressed. Thereafter, the methodology replicated that used by Lawson et al. [1]. Firstly, participants were asked to sketch a plan layout of all rooms on all floors of their house. This provided a visual reminder to the participants, who were required to consider the layout during the trial. It also familiarized the experimenter with the layout, which would help them understand comments made by participants. Then, participants were asked to imagine that it was the middle of the night and that they, and everyone else who typically sleeps in their house, were asleep in their beds. They were asked what actions they would take if they were woken by a faint crackling noise coming from the kitchen. They were told that this noise was caused by a fire if their anticipated action sequence led them to approach the kitchen. They were told to be reasonably explicit, and were probed for more detail if not enough was given. Every act reported was recorded, in order, until they were told to stop, which was typically when they had exited their house, or reached a state that would remain unchanged until the fire brigade arrived. The reported acts were recorded on a laptop and displayed on a projection screen so that participants could see their predicted act sequences. The sketches of the floor plans were also recorded by the experimenter.

As in the initial investigation into this predictive approach [1], the reported actions were coded against a common taxonomy of human behavior in fire, taken from Canter et al. [2]. Every effort was made to map the reported acts onto the taxonomy, but if this was not possible, they were mapped onto categories generated from the Lawson et al. study [1], or new categories were created. The frequencies with which each action occurred were recorded.

A matrix was then created in which the number of times each act followed every other act was recorded. The matrix was used to generate standardized residuals (observed frequency minus expected frequency ${ }^{1}$, divided by square root of expected frequency) for each transition between groups of related acts. Canter et al. [2] reported the standardized residuals as "strength of association" values and used them to provide information on the relationships between acts.

\footnotetext{
${ }^{1}$ Expected frequency $=($ row total $*$ column total $) /$ grand total
} 


\section{Results}

The taxonomy of acts, and frequencies with which each act was reported is shown in Table 1. This shows the frequency with which each of the acts from the taxonomy were reported in this study. The last column shows the values from the study of behavior in real fires [2]. Note that acts 1-25 were from the original taxonomy [2], acts $27 \mathrm{a}, 27 \mathrm{c}, 28 \mathrm{a}$ and $39 \mathrm{a}$ were found in the first predictive study [1], and acts 42a and $43 \mathrm{a}$ were found in this study, but could not be mapped to any previous act. Only two acts were removed from the analysis: "collect mobile/cordless phone" and "turn burglar alarm off'. These were removed as the prevalence of these technologies is likely to have increased since the original study in 1980, and therefore the comparison of these tasks would be unrepresentative.

Table 1. Taxonomy of acts against which the actions reported in this experiment were coded.

\begin{tabular}{|c|c|c|c|}
\hline \multirow[b]{2}{*}{ Code } & \multirow[b]{2}{*}{ Action category } & \multicolumn{2}{|c|}{ Frequency } \\
\hline & & $\begin{array}{l}\text { This study: } \\
20 \text { participants }\end{array}$ & $\begin{array}{l}\text { Canter et al. [2]: } \\
41 \text { participants }\end{array}$ \\
\hline $1 \mathrm{a}$ & Pre-event actions & 0 & 42 \\
\hline $2 \mathrm{a}$ & Perception of stimulus (ambiguous) & 5 & 40 \\
\hline $2 b$ & Alerted/awoken (ambiguous) & 2 & 20 \\
\hline $2 c$ & Note behaviour of others (ambiguous) & 0 & 16 \\
\hline $3 a$ & Perception of stimulus (associated with fire) & 2 & 12 \\
\hline $3 b$ & Note fire (development) & 20 & 31 \\
\hline $3 \mathrm{c}$ & Encounter smoke & 0 & 35 \\
\hline $4 \mathrm{a}$ & Interpretation (incorrect) & 4 & 23 \\
\hline $4 \mathrm{~b}$ & Disregard/ignore prior stimulus & 1 & 15 \\
\hline $5 \mathrm{a}$ & Receive warning/information/instruction & 3 & 73 \\
\hline $5 b$ & Ask advice/request information & 5 & 16 \\
\hline $6 a$ & Search for people (in smoke) & 0 & 18 \\
\hline $6 b$ & Encounter person in smoke & 0 & 12 \\
\hline $6 c$ & Check state of victim & 0 & 6 \\
\hline $7 \mathrm{a}$ & Observe rescue attempt & 0 & 19 \\
\hline $8 \mathrm{a}$ & Advise/instruct/reassure & 29 & 51 \\
\hline $8 b$ & Note agitated state of person & 0 & 5 \\
\hline $9 \mathrm{a}$ & Feel calm/unconcerned & 0 & 11 \\
\hline $10 \mathrm{a}$ & Experience negative feelings & 3 & 16 \\
\hline $10 \mathrm{~b}$ & Experience uncertainty & 1 & 15 \\
\hline $10 \mathrm{c}$ & Feel concern about occupants & 0 & 16 \\
\hline $10 \mathrm{~d}$ & Request assistance (urgent) & 0 & 2 \\
\hline $11 \mathrm{a}$ & Fire equipment faulty/unable to work & 0 & 8 \\
\hline $11 b$ & Struggle with environment & 0 & 5 \\
\hline $12 \mathrm{a}$ & Seek information/investigate & 52 & 76 \\
\hline $13 \mathrm{a}$ & Realize door to fire area open & 0 & 6 \\
\hline $13 b$ & Prevent fire spread & 13 & 29 \\
\hline $13 \mathrm{c}$ & Ensure accessibility & 2 & 10 \\
\hline $14 \mathrm{a}$ & Indirect involvement in activity & 0 & 27 \\
\hline $14 b$ & Wait for person/action to be completed & 2 & 22 \\
\hline $15 \mathrm{a}$ & Rescue & 0 & 42 \\
\hline
\end{tabular}




\begin{tabular}{llll}
\hline & & \multicolumn{2}{c}{ Frequency } \\
Code & Action category & This study: & Canter et al. [2]: \\
& & 20 participants & 41 participants \\
\hline 16a & Go/gain access to house with fire & 8 & 40 \\
16b & Go to neighbour's house & 5 & 12 \\
17a & Dress, gather valuables & 21 & 20 \\
$18 \mathrm{a}$ & Fetch things to fight fire with & 23 & 22 \\
18b & Fight fire & 28 & 25 \\
19a & Evasive & 9 & 33 \\
19b & Leave immediate area & 1 & 51 \\
20a & Forced back by/breathing difficulties/due to & 0 & 48 \\
& smoke/flames & & \\
20b & Cope with smoke & 1 & 15 \\
20c & Struggle through smoke & 0 & 8 \\
20d & Injured & 0 & 6 \\
21a & Pass through/enter fire area (investigate etc) & 8 & 35 \\
22a & Warn & 5 & 34 \\
22b & Phone for assistance & 18 & 7 \\
23a & Rescued/assisted & 1 & 13 \\
23b & Rescued from window & 0 & 4 \\
24a & Note/wait for fire brigade arrival & 16 & 45 \\
25a & Enter area of minimal risk & 23 & 52 \\
27a & Take/carry pet & 4 & 0 \\
27c & Call for pets & 1 & 0 \\
28a & Return to bedrooms & 16 & 0 \\
39a & Wake someone & 12 & 0 \\
42a & Take weapon/threaten/attempt to scare & 4 & 0 \\
43a & Move car & 1 & 0 \\
\hline & & & \\
& & & \\
\end{tabular}

The frequency of predicted acts demonstrated a significant relationship with Canter et al's [2] study of behavior in real fires (Spearman's rho: 0.323, N=55, $\mathrm{p}<0.05$ ). A significant relationship was also reported in Lawson et al. [1], albeit with a reduced set of tasks. These findings indicate that for this scenario, the predictive approach reliably demonstrates comparability for the frequency of acts with those reported by people who have been involved with real fires.

The transitions analyzed as part of this investigation are shown in Figure 1, which includes all transitions reported for domestic fires by Canter et al. [2]. Each labeled node represents a group of actions from Table 1. The arrows represent transitions between the nodes, by pointing to subsequent acts. There is no meaning in the position of the act nodes. The standardized residuals for each of the labeled arrows from this study and Canter et al. [2] are shown in Table 2.

The standardized residuals demonstrated a significant relationship between this study, and those reported in Canter et al. [2] (Spearman's rho: 0.340, N=37, $\mathrm{p}<0.05$ ). A significant finding was also reported by Lawson et al. [1], again indicating repeatability in the approach, and (in this instance) comparability to behavior in real fires. 


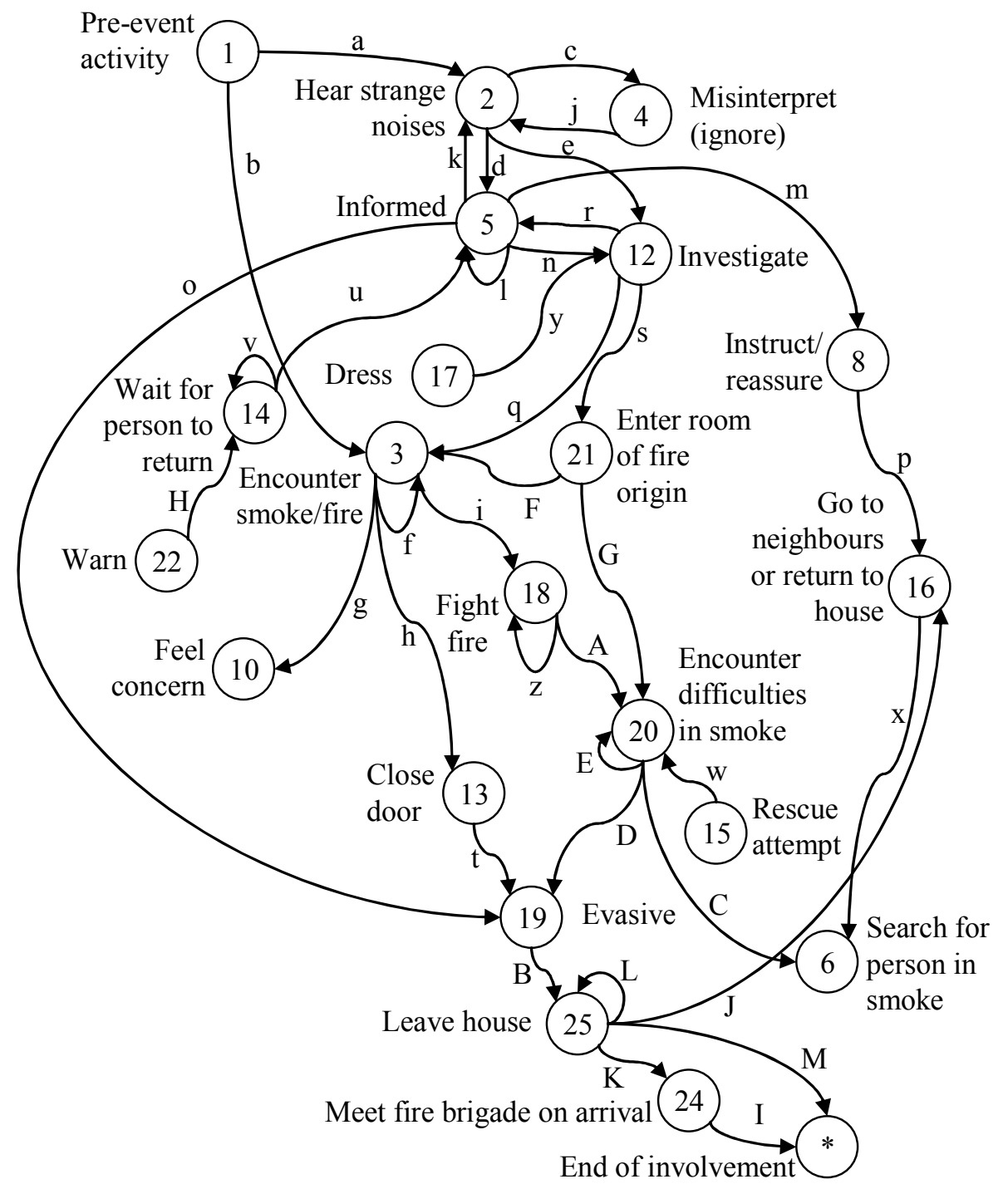

Fig. 1. Transitions investigated as part of this study.

Table 2. Standardized residuals for the transitions.

\begin{tabular}{lll}
\hline Transition & This study & Canter et al. [2] \\
\hline a & 0 & 9.84 \\
b & 0 & 1.34 \\
c & 3.64 & 6.26 \\
d & -0.39 & 0.97
\end{tabular}




\begin{tabular}{|c|c|c|}
\hline Transition & This study & Canter et al. [2] \\
\hline $\mathrm{e}$ & 0.06 & 5.81 \\
\hline$f$ & -1.23 & 2.12 \\
\hline g & 1.38 & 1.34 \\
\hline h & 0.95 & 0.89 \\
\hline $\mathrm{i}$ & 0.79 & 0.00 \\
\hline $\mathrm{j}$ & -0.18 & 4.02 \\
\hline $\mathrm{k}$ & -0.22 & 0.89 \\
\hline 1 & 1.97 & 2.46 \\
\hline $\mathrm{m}$ & 0.36 & 1.34 \\
\hline $\mathrm{n}$ & 0.89 & 0.97 \\
\hline o & -0.45 & 1.72 \\
\hline $\mathrm{p}$ & -0.10 & 0.45 \\
\hline$q$ & 5.59 & 8.88 \\
\hline $\mathrm{r}$ & -0.11 & 0.55 \\
\hline $\mathrm{s}$ & 1.52 & 3.13 \\
\hline $\mathrm{t}$ & 1.10 & 0.45 \\
\hline $\mathrm{u}$ & 4.56 & 2.68 \\
\hline $\mathrm{v}$ & -0.11 & 0.89 \\
\hline $\mathrm{w}$ & 0.00 & 1.79 \\
\hline $\mathrm{x}$ & 0.00 & 0.45 \\
\hline $\mathrm{y}$ & 1.88 & 1.79 \\
\hline $\mathrm{z}$ & 5.66 & 3.58 \\
\hline A & 2.13 & 1.79 \\
\hline B & 1.75 & 14.30 \\
\hline $\mathrm{C}$ & 0.00 & 0.89 \\
\hline $\mathrm{D}$ & -0.16 & 3.90 \\
\hline $\mathrm{E}$ & -0.06 & 3.58 \\
\hline $\mathrm{F}$ & 3.30 & 2.24 \\
\hline G & -0.16 & 1.34 \\
\hline $\mathrm{H}$ & -0.35 & 0.89 \\
\hline $\mathrm{I}^{2}$ & - & 9.39 \\
\hline $\mathrm{J}$ & 3.28 & 4.47 \\
\hline K & 5.61 & 4.47 \\
\hline $\mathrm{L}$ & -1.23 & 4.47 \\
\hline $\mathrm{M}^{2}$ & - & 2.24 \\
\hline
\end{tabular}

The findings were investigated further to identify any opportunities to improve the approach through the next stages of its development. A scatter plot in Figure 2 shows the standardized residuals from this study plotted against those from Canter et al [2].

2 Note "End of involvement" was not listed in the original taxonomy of acts, and therefore strength of association values were not calculated for acts leading to this one 
The dashed line indicates a theoretical perfect correlation. A visual inspection indicates that there were under-representations of the transitions $\mathrm{B}$, a, and $\mathrm{L}$ in this study. These relate to: "evasive" to "leave house"; "pre-event activity" to "hear strange noises"; and "leave house" to "leave house". Possible reasons for these under-representations are made in the discussion.

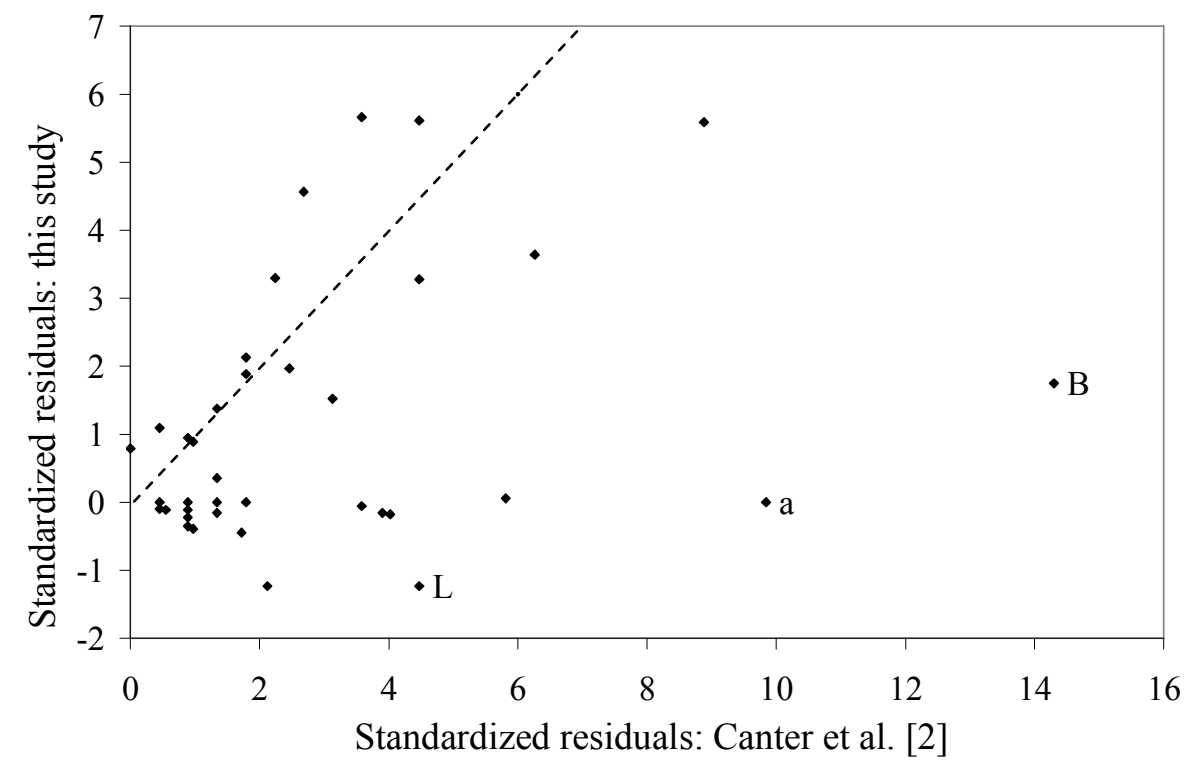

Fig. 2. Strength of association values for the transitions from each of the studies. A theoretical perfect correlation is indicted (dashed line).

\section{Discussion}

The results demonstrated that in this scenario, the approach used yielded a significant relationship between the frequencies and sequences of actions participants predicted they would take in a domestic fire when compared to a study of interviews with survivors of real domestic fires. These significant relationships were also identified in the initial application of this approach [1]. The significant relationships for the frequency and sequence of acts for both predictive studies indicate that, in this instance, the approach demonstrated reliability, and comparability with one study of human behavior in a real fire. These findings imply that with development the approach could be applied to reveal what actions people will take in an emergency situation. It must be emphasized that further work is required - for example an investigation into generalizability to a range of different scenarios. However, once developed, and if proven to meet all the criteria of a quality method, it could be used to predict behavior in novel situations as determined by the scenario presented to the participants. The resources required are low (a laptop computer, projector and 
whiteboard, and 20 participants) and it does not require any physical or virtual creation of an environment, only a brief written description of the scenario of interest.

While this study demonstrated significant relationships for the sequence of actions, Fig 2 revealed that some tasks were under-represented in this approach, and that enhancements may further improve the validity. "Evasive" to "leave house" (B) might have been infrequently observed due to differences in interpretation of participants' responses to Canter et al. [2]. For example, participants in this study stated where they would go to e.g. "I would get out of the house". This was never interpreted as "I would put distance between me and the fire" and then "I would get out of the house", although this sequence was identified more frequently in the Canter et al. study [2]. In the next stage of the development of this approach, the interviewing will probe for more detail on participants' predicted locations.

The sequence related to "pre-event activity" (a), would also have been expected to be under-represented, as the participants were told that they heard a fire, and therefore their first reported action was never anything which could be classified as a "preevent activity". For subsequent trials, it is recommended that the approach is altered such that pre-event activities (and "end of involvement") are recorded as acts.

The final sequence which also shows apparent under-representation is L "leave house" to "leave house". This group actually includes the sub-category of "enter area of minimal risk". Therefore, if a participant predicts that they would move out of their house, then move to another safe place, this would increase the occurrence of this transition. This also may be realized through greater attention to reporting locations.

It is worth noting that significant relationships were found for the sequence and frequency of acts despite a stricter analysis procedure than that used in Lawson et al. [1]. In this development study, only tasks relating to changes in technology since 1980 were removed, whereas in the initial predictive study several acts were removed which were not anticipated due to the experimental protocol or scenario. This change was made to reflect more accurately the anticipated end-use of the approach. A Human Factors practitioner would not know which tasks were unexpected due to the experimental protocol, and therefore this analysis is more representative of how the approach will be implemented to predict behaviors.

\section{Conclusions}

This study was conducted to investigate developments on an approach for predicting human behavior in an emergency situation, in which participants were asked what actions they would take if they experienced a domestic fire in their house at night. The frequency and sequence of acts reported were compared to a study of human behavior in real fires. A significant relationship was found for both, as they were in an initial study of the predictive approach, indicating reliability. This is despite a stricter analysis procedure, which excludes far fewer tasks. Recommendations have been made to improve the methodology, mainly asking participants for more detail about their locations, which will be implemented in the next phase of the development of this approach. 
The approach continues to show promise as a low resource method, which (with development) could be used as part of the Human Factors professional's toolkit for predicting behavior in novel situations. It does not require a physical or virtual mockup, and does not put participants in any danger.

\section{Citations}

1. Lawson, G., Sharples, S., Cobb, S., Clarke, D.: Predicting the Human Response to an Emergency. Accepted for publication in: Bust, P.D. (ed.) Contemporary Ergonomics. Taylor and Francis, London (in press).

2. Canter, D., Breaux, J., Sime, J.: Domestic, Multiple Occupancy, and Hospital Fires. In: Canter, D. (ed.) Fires and Human Behaviour. pp. 117-136, John Wiley and Sons, New York (1980)

3. Laughery, R.: Simulation and Modelling as a Tool for Analysing Human Performance. In: Wilson, J.R., Corlett, N. (eds.) Evaluation of Human Work ( $3^{\text {rd }}$ Edition), pp. 219-238. Taylor and Francis, London (2005)

4. Hamilton, W.I., Clarke, T.: Driver Performance Modelling and its Practical Application to Railway Safety. Applied Ergonomics. 36, 661-670 (2005)

5. DiFac IST5-035079, http://difac.net

6. Lawson, G., D'Cruz, M., Bourguignon, D., Pentenrieder, K.: Training in the Digital Factory. Paper Presented at the IFAC Workshop on Manufacturing, Modelling, Management and Control, Budapest. (2007)

7. Purser, D.A., Bensilum, M.: Quantification of Behaviour for Engineering Design Standards and Escape Time Calculations. Safety Science. 38, 157-182 (2001)

8. Brooks, C.J., Muir, H.C., Gibbs, P.N.G.: The Basis for the Development of a Fuselage Evacuation Time for a Ditched Helicopter. Aviation, Space and Environmental Medicine. $72,553-561(2001)$

9. Deere, S.J., Galea, E.R., Lawrence, P.J.: A Systematic Methodology to Assess the Impact of Human Factors in Ship Design. Applied Mathematical Modelling. 33, 867-883 (2009)

10.Dane, F.C: Research Methods. Brooks/Cole Publishing Co, Belmont (1990)

11.Silverman, B.G., Johns, M., Cornwell, J., O’Brien, K.: Human Behavior Models for Agents in Simulators and Games: Part I - Enabling Science with PMFserv. Presence: Teleoperators and Virtual Environments, 139-162 (2006)

12.Kirwan, B., Ainsworth, L.K.: A Guide to Task Analysis. Taylor and Francis, London (1992)

13.Bakeman, R., Gottman, J.M.: Observing Interaction: An Introduction to Sequential Analysis. Cambridge University Press, Cambridge (1986)

14.Wilson, J.R.: Methods in the Understanding of Human Factors. In: Wilson, J.R., Corlett, N. (eds.) Evaluation of Human Work ( $3^{\text {rd }}$ Edition), pp. 1-31. Taylor and Francis, London (2005)

Acknowledgements. The authors acknowledge Professor David Canter for permission to use the adapted material from Domestic, Multiple Occupancy, and Hospital Fires [2]. 